\title{
CDMA-Based Analog Network Coding for Underwater Acoustic Sensor Networks
}

\author{
Hovannes Kulhandjian, Member, IEEE, Tommaso Melodia, Member, IEEE, and \\ Dimitrios Koutsonikolas, Member, IEEE
}

\begin{abstract}
We propose ANC-CDMA, a CDMA-based analog network coding scheme for underwater acoustic sensor networks (UW-ASNs) that can significantly improve the underwater acoustic channel utilization. First, we analyze a unidirectional multihop network, in which two acoustic nodes separated by two hops are assigned the same code-division channel (i.e., spreading code) to transmit concurrently. The packets transmitted by the two nodes will collide at the intermediate (relay) node. However, we show that, by exploiting a priori information, i.e., the interfered packet previously received from one of the nodes, and applying joint channel estimation through pilot supervision along with a newly designed adaptive RAKE receiver, the relay node can cancel the interference before decoding the packet of interest. We then extend our analysis to a bidirectional network, in which two nodes first concurrently transmit their packets to the relay using the same spreading code. The relay amplifies and forwards the received interfered packet to the two nodes. We show that either node can still decode the packet of interest after equalizing the channel effects introduced during the propagation from the relay to itself and jointly estimating multipath affected channels and suppressing the self-interference signal before applying the adaptive RAKE receiver. The proposed ANC-CDMA scheme is implemented in a testbed based on Teledyne Benthos Telesonar SM-975 underwater modems and tested extensively in Lake LaSalle. Experiments and simulations demonstrate that for a 1-2 dB tradeoff in signalto-noise ratio (SNR), the proposed scheme can significantly improve the channel utilization of a unidirectional and bidirectional networks by up to $50 \%$ and $100 \%$, respectively, compared with conventional multiuser DS-CDMA scheme.
\end{abstract}

Index Terms-Underwater acoustic communications, UW-ASNs, analog network coding, ANC, PNC, DS-CDMA, interference cancelation, joint channel estimation, RAKE-matched-filter.

Manuscript received March 6, 2014; revised September 25, 2014, January 15, 2015, and May 9, 2015; accepted June 26, 2015. Date of publication July 13, 2015; date of current version November 9, 2015. This work was supported in part by the U.S. National Science Foundation under Grants CNS1055945 and CNS-1126357. An earlier version of this paper was presented at the ACM International Conference on UnderWater Networks and Systems, Los Angeles, CA, USA, November 2012 [1]. The associate editor coordinating the review of this paper and approving it for publication was A. Zajic.

H. Kulhandjian is with the Department of Electrical and Computer Engineering, California State University, Fresno, CA 93740 USA (e-mail: hkulhandjian@csufresno.edu).

T. Melodia is with the Department of Electrical and Computer Engineering, Northeastern University, Boston, MA 02115 USA (e-mail: melodia@ece. neu.edu).

D. Koutsonikolas is with the Department of Computer Science and Engineering, University at Buffalo, The State University of New York, Buffalo, NY 14260 USA (e-mail: dimitrio@buffalo.edu).

Color versions of one or more of the figures in this paper are available online at http://ieeexplore.ieee.org.

Digital Object Identifier 10.1109/TWC.2015.2456012

\section{INTRODUCTION}

$\mathbf{U}$ NDERWATER acoustic sensor networks (UW-ASNs) have attracted considerable attention recently [2]-[5]. Although radio frequency (RF) electromagnetic and optical waves are the dominant physical communication carriers in terrestrial wireless communications, in water they are severely affected by high attenuation and scattering, respectively. Acoustic communication is therefore the transmission technology of choice for underwater networked systems [3].

The underwater acoustic (UW-A) channel is known to suffer from limited bandwidth and spectral efficiency. The recently proposed physical layer network coding (PNC) [6] and analog network coding (ANC) [7] schemes have demonstrated significant improvements in spectral efficiency and throughput gains under several network scenarios. The basic idea of PNC and ANC is to better utilize the wireless bandwidth by allowing concurrent transmissions of signals over the wireless medium so that they intentionally interfere with each other. The receiver, having knowledge of the interfered signal from prior transmissions, will suppress it and be able to detect the information of interest.

The objective of this paper is to explore the idea of ANC, which is essentially a form of linear self-interference cancellation with the use of a priori information [8], in multi-hop UWASNs. The idea of linear self-interference cancellation was first introduced in [9] and over the years it has evolved in different forms [8], [10]-[13].

Unlike in terrestrial wireless communications, in UW-A communications multipath is prevalent [14]. Therefore, designing a receiver tailored for ANC that extracts the information of interest from the two interfered signals in severely affected multipath channels is very challenging.

We therefore introduce ANC-CDMA, a CDMA-based ANC scheme for multi-hop unidirectional and bi-directional UWASNs. We consider a direct-sequence code-division multipleaccess (DS-CDMA) scheme. ${ }^{1}$

The main contributions of this paper can be outlined as follows.

1) For the first time, we are proposing a new CDMA-based ANC, ANC-CDMA, for environments affected by severe multipath, including underwater acoustic channels.

\footnotetext{
${ }^{1} \mathrm{CDMA}$ is one of the most promising physical layer and multiple access techniques for UW-ASNs [3], since it is robust to frequency-selective fading and can compensate for the effect of multipath through RAKE receivers [4].
} 
2) We first analyze a unidirectional multi-hop network, in which two nodes, separated by two hops, are assigned the same spreading code to communicate concurrently. The packets transmitted by the two nodes will collide at the intermediate (relay) node. We show that, by exploiting a priori information, i.e., the interfered packet previously received from one of the nodes, and jointly estimating the two multipath Rayleigh fading channels through pilot supervision, the relay node can cancel the interference. We address two cases, namely synchronous and asynchronous packet arrival at the relay. We design a new adaptive RAKE receiver to be used by the relay to decode the packet of interest.

3) We then extend our analysis to a more challenging case, a bi-directional network, in which Alice and Bob first concurrently transmit their packets to the relay using the same spreading code. The relay node inserts a new set of pilot bits in the received packet, then amplifies and forwards it to Alice and Bob. Although the two packets will again interfere at the relay node, we show that Alice/Bob can still decode the packet of interest. To retrieve Alice's packet, Bob will first adaptively equalize the channel effects introduced during the propagation from the relay to itself. Then he will jointly estimate the multipath affected channels and suppress the self-interference signal before applying the proposed adaptive RAKE receiver.

4) We finally discuss the first implementation of ANCCDMA in a real testbed based on Teledyne Benthos [15] Telesonar SM-975 underwater modems. We conduct simulations and testbed experiments in a lake to evaluate the performance of the proposed scheme in terms of bit error rate (BER) and compare it with conventional multiuser DS-CDMA. Performance evaluation results reveal that the proposed scheme performs close to conventional multi-user DS-CDMA in terms of BER for a given SNR. Moreover, at the price of $1-2 \mathrm{~dB}$ increase in SNR, it can achieve a throughput gain of up to $50 \%$ and $100 \%$ for unidirectional and bi-directional networks, respectively, for a target BER level.

The rest of this paper is organized as follows. We first discuss related work in Section II. In Section III, we introduce the unidirectional and bi-directional ANC-CDMA schemes. In Section IV, we describe the system model with detailed discussions on equalization, joint channel estimation, and receiver design. In Section V, we evaluate the proposed scheme through simulation and testbed experimentations. Finally, in Section VI, we draw the main conclusions.

Notation: The following notation is used throughout the paper. Boldface lower-case letters indicate column vectors, boldface upper-case letters indicate matrices, $\mathbf{x}^{H}$ denotes the Hermitian of vector $\mathbf{x}, \mathbf{I}_{N}$ and $\mathbf{0}_{N}$ are the identity and zero matrices of dimensions $N \times N$ respectively, $\operatorname{tr}\{\mathbf{X}\}$ represents the trace of a matrix $\mathbf{X}, \mathbb{E}\{\cdot\}$ represents statistical expectation, $\|$. $\|$ is the Euclidean norm of a vector, $\mathfrak{R e}(\cdot)$ denotes the real part of a complex valued vector, $\bmod (\cdot)$ denotes modular arithmetic, $\lfloor\cdot\rfloor$ is the floor function and $\operatorname{sgn}(\cdot)$ denotes zerothreshold quantization.

\section{RELATED WORK}

There are only a handful of works that have attempted to propose ANC schemes tailored for frequency-selective fading channels. In [16], an orthogonal frequency-division multiplexing (OFDM)-based two-slot pilot-assisted channel estimation and receiver design is proposed for bi-directional ANC. In the first time slot, the two users simultaneously transmit their supervised pilot bits to the relay with one of the pilot signals cyclically shifted to enable the relay to separate and estimate the channel state information (CSI) from both users. In the second time slot, the relay broadcasts another set of pilot bits to the two users. The authors assume that the CSIs, estimated by the relay, will be sent via an ideal feedback channel, which might not be very practical in UW-A communications, as the CSIs might change by the time they arrive at the receiver. Due to the long propagation delays in UW-A channel it is also inefficient to allocate two separate time slots for pilot signal transmissions. Moreover, the CSIs are estimated only once for a stream of data transmissions. However, due to the time varying nature of UW-A channel, it is essential to estimate the CSIs periodically [1]

In [17], three iterative receiver designs for OFDM modulated PNC in two-way relay assisted UW-A communications are proposed. Unlike in ANC, in which the relay amplifies and forwards the two received interfered signals, in PNC the relay decodes and forwards a network coded version of the two information bit streams. In [17], the authors assume that the relay node is equipped with an array of receiving hydrophones and the relay estimates the CSIs using the sparse channel estimator discussed in [18]. However, in [18] the authors address channel estimation for a single channel, while in PNC one needs to estimate the two channels jointly, which can be challenging. To estimate the interfered signal at the relay the authors propose iterative maximum a posteriori (MAP) and Gaussian message passing (GMP) estimation schemes. In addition to that, the authors focus on decoding and forwarding the interfered information at the relay node. Unlike [17], our work considers a CDMA scenario and we address the detection of information of interest at either node.

In [19], a PNC over frequency selective two-way relay channel is proposed, where two users exchange information in two phases through an intermediate relay node using the decode-and-forward approach. A list decoding algorithm and a minimum mean-square-error (MMSE) based detector are proposed to allow the relay to decode the XOR of the transmitted codewords. The authors assume that the channel is constant over the transmission of each data frame and the CSIs are known at the relay.

A few other works [20]-[22] have proposed CDMA-based PNC/ANC schemes. In [20], a two-phase multiuser two-way relay network is proposed, in which multiple pairs of users communicate with their preassigned users using a common intermediate relay node. In phase one, all pairs of users simultaneously transmit their information to the relay. In phase two, the relay transmits an estimate of the XORed symbol to each user pair. The authors assume flat fading channel and that the channel gains remain constant throughout the duration of the communication, which is usually not the case in UW-A 


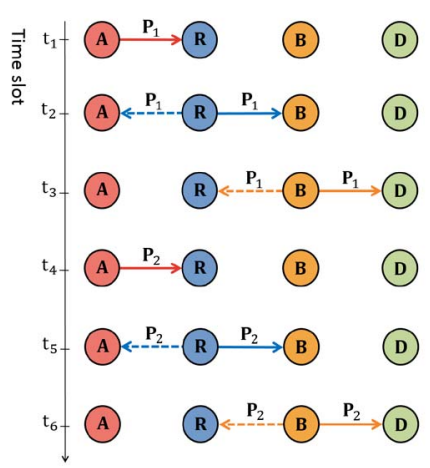

(a)

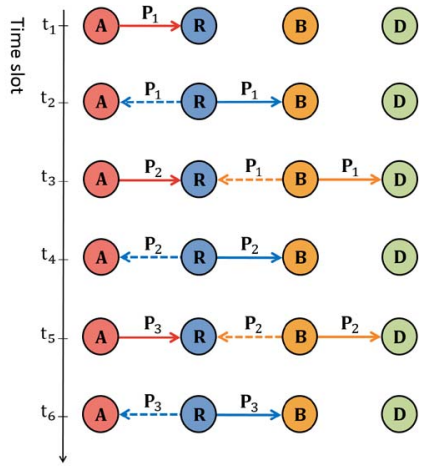

(b)
Fig. 1. Four-node chain network topology: (a) Conventional CDMA scheduling, (b) Unidirectional ANC-CDMA scheduling.

communications. Moreover, in this work the authors do not address the channel estimation problem.

Similarly, in [21] a CDMA-based ANC is proposed for two way relaying. After the relay receives the superimposed information from the two users in the first transmission round it amplifies and forwards the received packet in the next transmission round. MMSE filtering is applied to decode the information bits. In the proposed scheme, the authors do not address channel estimation and it is assumed that CSIs remain constant throughout the communication.

In [22], an ANC CDMA scheme is proposed where multiple pairs of users communicate via a shared intermediate relay each pair using the same spreading code. The authors assume there is no fading, which is not practical in an underwater scenario. In all of the above works [16], [17], [19]-[22] the authors assume perfect synchronous arrival of the two packets at the relay, which is very difficult to achieve in UW-A communications, due to the long propagation delays [3], [23]. Our proposed scheme, on the other hand, does not require synchronous arrival of the two packets.

\section{NeTWORK MOdels}

\section{A. Unidirectional Multi-Hop Linear Topology}

We first consider a wireless multi-hop linear network topology, shown in Fig. 1(a). In this scenario, a source (Alice), denoted by $A$, would like to transmit packets $P_{1}, P_{2}, \ldots$, through multi-hop routing, to the destination, $D$, using a unique spreading code previously assigned to it. It takes three time slots to convey a packet from the source to the destination. The solid line indicates the scheduled transmissions, while the dashed line is the interference generated due to the broadcast nature of wireless transmission. When packet $P_{1}$ is being forwarded by Bob denoted by $B$ in time slot $t_{3}, A$ should not use the same spreading code to transmit $P_{2}$, since $B$ 's transmission may cause interference to the relay, $R$. The average throughput and end-to-end delay of this network will be improved if we allow concurrent transmissions by two nodes located two hops away, e.g., $A$ and $B$. Concurrent transmissions will allow $A$ to transmit $P_{2}$, while $B$ is forwarding $P_{1}$ to the destination, $D$, in time slot $t_{3}$ using the same spreading code. The two packets, $P_{1}$ and $P_{2}$

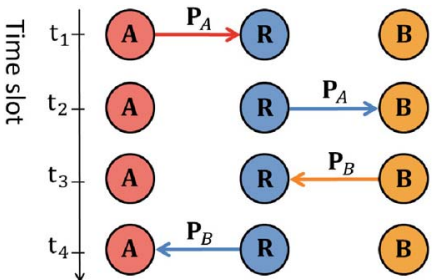

(a)

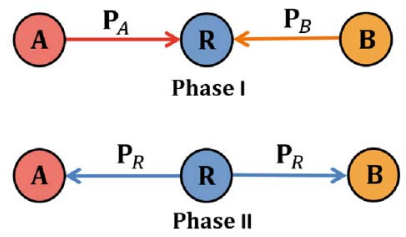

(b)
Fig. 2. Alice-relay-Bob topology: (a) Conventional CDMA scheduling, (b) Bi-directional ANC-CDMA scheduling.

will interfere with each other at $R$. However, using a priori information, $P_{1}$, from time slot $t_{2}, R$ will cancel the interference generated by $B$, before decoding $P_{2}$ and forwarding it to $B$ in the next time slot. The proposed (ANC-CDMA) scheduling scheme for a unidirectional network is shown in Fig. 1(b). Under this scheduling scheme the wireless channel is further exploited, since no additional time slot is required to transmit the packet, $P_{2}$, from $A$ to $R$. By utilizing the ANC-CDMA scheduling scheme on average only two time slots are needed to transmit a packet from source to destination using a single spreading code. The performance gain of a scheduling scheme can be expressed by the transmission efficiency, defined in [13] as the ratio of the time taken for the transmission of $P$ packets under the conventional scheduling scheme and the scheduling scheme employing overlapped transmissions, respectively. The transmission efficiency, $\Gamma_{4}$, for the four-node scenario presented above is given by

$$
\Gamma_{4}=\frac{3 P}{2(P-1)+3} \approx \frac{3}{2}, P \gg 1,
$$

where $P$ is the total number of packets transmitted by $A$. Equivalently, for a linear network topology with $N(N \geq 4)$ nodes, as shown in [13], the same gain is achieved for $P \gg 1$.

We observe that utilizing the proposed scheme in a multihop chain topology the efficiency of the unidirectional linear network can be improved by up to $50 \%$ over the conventional scheduling scheme.

\section{B. Bi-Directional Alice-Relay-Bob Topology}

We now consider a wireless bi-directional linear network, shown in Fig. 2(a). In this scenario, Alice and Bob would like to transmit packets $P_{A}$ and $P_{B}$ respectively to each other through the help of a relay node, as they cannot hear each other. Employing conventional scheduling schemes, it takes four time slots to exchange the packets using a single spreading code.

The average throughput and end-to-end delay of this network will be improved significantly if we allow Alice and Bob to transmit $P_{A}$ and $P_{B}$ concurrently to the relay. The relay amplifies the interfered packet and forwards it to both Alice and Bob. Although the packets will interfere with each other at the relay node, as Alice and Bob are utilizing the same spreading code, we show that either user can still decode the packet of interest by first estimating and compensating the channel effects and cancelling self-interference from the received packet. The proposed ANC-CDMA scheme for bi-directional network is 
shown in Fig. 2(b). Under this scheduling scheme the wireless channel is further exploited and only two transmission slots are required instead of four, which in theory leads to channel utilization gain of $100 \%$ over the conventional scheduling scheme.

\section{System Model And PRoblem Formulation}

\section{A. Unidirectional CDMA-Based ANC}

We first consider a CDMA-based unidirectional network shown in Fig. 1(b), in which both Alice and Bob would like to access the medium in time slot $t_{3}$ using the same spreading code and concurrently transmit packets $P_{A}$ and $P_{B}$ to the relay and destination, respectively.

Because of the highly frequency-selective distortion caused by multipath propagation in the UW-A channel, it is essential to estimate the CSI periodically [1]. One possible way to estimate both channels is to let Alice and Bob transmit a set of pilot bits, $N_{p}$, at different time slots before their data packet transmission. However, due to the long propagation delays in UW-A channel using such an approach might not be practical. First, this would require long guard-bands and perfect synchronization, which is difficult to achieve in underwater acoustic channels. Second, due to the highly time varying nature of the UW-A channel it might be hard to capture the channel variations throughout data packet transmission. The channel conditions may change before the actual data packet arrives at the receiver node. Accordingly, we utilize a set of $N_{p}$ pilot bits that are repeated periodically and are inserted in each packet distanced less than the coherence time, $T_{C T}$, of the channel, i.e., each set of $N_{p}$ pilot bits are repeated every $T_{C T}$ period. The pilot bits will be used to jointly estimate the CSIs from Alice-to-relay and Bob-to-relay.

The baseband supervised and information bits transmitted by Alice and Bob are

$$
\begin{array}{ll}
\mathbf{x}_{A}(i)=b_{A}(i) \sqrt{E_{A}} \mathbf{s}, \quad i=1,2, \ldots, \\
\mathbf{x}_{B}(i)=b_{B}(i) \sqrt{E_{B}} \mathbf{s}, \quad i=1,2, \ldots,
\end{array}
$$

where $\mathbf{s} \in \frac{1}{L}\{ \pm 1\}^{L}$ denotes the normalized spreading code of length $L$ used by Alice and Bob, $E_{A}$ and $E_{B}$ denote the transmit energy per bit, $b_{A}(i), b_{B}(i) \in\{-1,1\}$ are $i^{\text {th }}$ pilot/information bits binary phase-shift-keying (BPSK) data modulated and transmitted by Alice and Bob, respectively. The information bits $b_{k}(i)$ are viewed as binary equiprobable random variables that are independent across the users $(k=A, B)$ and within a user steam $(i=1,2, \ldots)$ [24].

Before transmission of the information bits, each chip of the spreading sequence is multiplied by a pulse shaping signal $p(t)$ and a carrier. The normalized modulated spreading sequence in the time domain is then denoted by

$$
s(t)=\frac{1}{L} \sum_{l=1}^{L} d(l) p\left(t-l T_{c}\right) e^{j 2 \pi f_{c} t}
$$

where $d(l) \in\{-1,1\}$ is the $l^{\text {th }}$ transmitted chip of the spreading sequence, $T_{c}=T / L$ is the chip period, $T$ is the information bit duration, and $f_{c}$ is the carrier frequency.
We assume that packets propagate over multipath Rayleigh fading UW-A channels. The proposed scheme does not require synchronous arrival of the two packets at the relay, which is difficult to achieve due to long propagation delays in UW-A channels. However, for ease of exposition, we first address the synchronous arrival of the two packets and assume that packets transmitted by Alice and Bob experience the same number of resolvable multipaths at the relay, i.e., $M_{A R}=M_{B R}=M$. Later, in Section IV-A2, we address the asynchronous case with different number of resolvable multipaths arrivals at the relay. In Section V-B, we discuss how to estimate the number of multipath components in practice. A preamble and a postamble, comprised of three identical chirp signals of duration $100 \mathrm{~ms}$ sweeping the bandwidth from $10 \mathrm{~Hz}$ to $5.12 \mathrm{kHz}$, each separated by a $50 \mathrm{~ms}$ guard-band are appended to each packet and are used for channel probing, symbol synchronization for chip-matched-filtering, and multipath delay spread estimation. The chirp signals are in baseband and are modulated by multiplying by a carrier $f_{c}$ before transmission. Considering a payload size $=1.25 \mathrm{kBytes}$ and spreading code length $L=32$, discussed later in Section $\mathrm{V}$, the overhead from the postamble is $<0.25 \%$, which is certainly low compared to the throughput gain that ANC-CDMA can provide.

The total baseband received signal at the receiver is

$r(t)=\sum_{i} \sum_{k=1}^{K} b_{k}(i) \sum_{m=1}^{M} h_{k, m} \sqrt{E_{k}} s_{k}\left(t-i T-\frac{m}{B}-\tau_{k}\right)+n(t)$,

where $h_{k, m}$ is the channel coefficient corresponding to the $k^{\text {th }}$ user due to multipath branch $m$ of the frequency-selective slowly fading channel. The channel coefficients are modeled as independent zero-mean complex Gaussian random variables that remain constant within a bit interval, $n(t)$ is ambient noise and $\tau_{k}$ is the relative time delay of the user $k$. Multipath spread in shallow water acoustic channel is in the order of a few tens of chip intervals $T_{c}$ for $L=63$ [25], and since the signal is bandlimited to $B=1 / T_{c}$, the tap delay line channel mode has taps spaced at chip intervals $T_{c}$. Spreading codes of length longer than the multipath spread are selected.

1) Synchronous Packet Arrival With Same Number of Multipath: After carrier demodulation, chip-matched-filtering, and sampling at the chip rate over a multipath extended bit period of $L+M-1$ chips, where $M$ is the number of resolvable multipaths, the received signal, $\mathbf{r}_{R}(i) \in \mathbb{C}^{L+M-1}$, (that is, the noise-affected superimposed version of the $i^{t h}$ bits of Alice and Bob) at the relay node is denoted by

$$
\mathbf{r}_{R}(i)=\sqrt{E_{A}} \mathbf{S}_{A}(i) \mathbf{h}_{A R}+\sqrt{E_{B}} \mathbf{S}_{B}(i) \mathbf{h}_{B R}+\mathbf{n}(i), i=1,2, \ldots
$$

where

$$
\mathbf{h}_{A R}=\left[h_{A R}(1), h_{A R}(2), \ldots, h_{A R}(M)\right]^{H}
$$

and

$$
\mathbf{h}_{B R}=\left[h_{B R}(1), h_{B R}(2), \ldots, h_{B R}(M)\right]^{H},
$$


are multipath channel coefficients from Alice-to-relay and Bob-to-relay of lengths $M . h_{A R}(q)$ and $h_{B R}(q)$ represent the $q^{\text {th }}$ resolvable path coefficients modeled as complex Gaussian random variables (Rayleigh-distributed) that remain constant during $T_{C T}$ block length (i.e., quasi-static fading).

$$
\begin{aligned}
& \mathbf{S}_{A}(i)=\mathbf{S}_{A}^{0}(i)+\mathbf{S}_{A}^{+}(i)+\mathbf{S}_{A}^{-}(i), \\
& \mathbf{S}_{B}(i)=\mathbf{S}_{B}^{0}(i)+\mathbf{S}_{B}^{+}(i)+\mathbf{S}_{B}^{-}(i),
\end{aligned}
$$

where

$$
\mathbf{S}_{k}^{0}(i)=b_{k}(i)\left[\begin{array}{cccc}
s(1) & 0 & \cdots & 0 \\
\vdots & s(1) & \ddots & \vdots \\
s(L) & \vdots & \ddots & 0 \\
0 & s(L) & & s(1) \\
\vdots & \vdots & \ddots & \vdots \\
0 & 0 & \cdots & s(L)
\end{array}\right]_{(L+M-1) \times M}
$$

$\mathbf{S}_{k}^{+}(i)=b_{k}(i+1)\left[\begin{array}{cccc}0 & \cdots & 0 & 0 \\ \vdots & \ddots & \vdots & \vdots \\ 0 & & 0 & 0 \\ s(1) & \ddots & \vdots & 0 \\ \vdots & \ddots & 0 & \vdots \\ s(M-1) & \ldots & s(1) & 0\end{array}\right]_{(L+M-1) \times M}$
The matrices $\mathbf{S}_{k}^{0}(i), \mathbf{S}_{k}^{+}(i)$, and $\mathbf{S}_{k}^{-}(i) \in \mathbb{R}^{(L+M-1) \times M}$ correspond to the spreading code matrices generated due to the transmission of bits $b(i), b(i+1)$, and $b(i-1)$, respectively, by user $k \in\{A, B\}$, Alice or Bob in this case. Note that Alice and Bob can reconstruct $\mathbf{S}_{A}(i)$ and $\mathbf{S}_{B}(i)$, respectively, using the pilot bits.

Lastly, $\mathbf{n}$ is the ambient noise in the underwater communication channel, which can be either natural or man-made [3]. The contributions of the major noise sources can be expressed through empirical formulae [26], [27], which provide power spectral densities of each source relative to frequency $f[\mathrm{kHz}]$ in $\left[\mathrm{dB}\right.$ re $\mu \mathrm{Pa}^{2}$ per $\left.\mathrm{Hz}\right]$

$$
10 \log N_{t}(f)=17-30 \log f
$$

$10 \log N_{s}(f)=40+20(s-5)+26 \log f-60 \log (f+0.03)$,

$10 \log N_{w}(f)=50+7.5 w^{1 / 2}+20 \log f-40 \log (f+0.4)$

$10 \log N_{t h}(f)=-15+20 \log f$, where $N_{t}, N_{s}, N_{w}, N_{t h}$ stand for turbulence, shipping, wind, and thermal noise, respectively. $s$ takes values between 0 and 1 , which corresponds for low and higher shipping activity, respectively. $w$ is the wind speed in $[\mathrm{m} / \mathrm{s}]$. The total noise power spectral density for a given frequency $f[\mathrm{kHz}]$ then is

$$
N(f)=N_{t}(f)+N_{s}(f)+N_{w}(f)+N_{t h}(f) .
$$

2) Asynchronous Packet Arrival With Different Number of Multipath: We now address the asynchronous arrival of the two packets at the relay with different number of multipaths. Without loss of generality, we assume Alice's packet arrives before Bob's with a relative time delay $\tau=\tau_{B}-\tau_{A}$, where $\tau_{A}$ and $\tau_{B}$ are the relative time delay from the first multipath component arrival of Alice's and Bob's packet, respectively and in this example $\tau_{A}=0$. In practice, the relative time delay, $\tau$, is estimated by chirp-matched-filtering the received signal and computing the time difference between the line-of-sight components of the two received packets. ${ }^{2}$ To formulate the problem, we round down the relative time delay to the nearest integer chip delay and define the discrete relative time delay as $\Delta=T_{c}\left\lfloor\frac{\tau}{T_{c}}\right\rfloor=d_{b} T+d_{c} T_{c}$, where $d_{b}=\left\lfloor\frac{\tau_{B}-\tau_{A}}{T}\right\rfloor$ and $d_{c}=\left\lfloor\frac{\tau_{B}-\tau_{A}}{T_{c}}\right\rfloor$ $\cdot \bmod (T)$ correspond to the number of bit and chip delays, respectively. Note that since the actual multipath, $h(t)$, is a continuous function of time, sampling the line-of-sight component of Bob's packet instead at the closest tap-delay-line location of Alice packet will not have much of an effect on our analysis. This is because we can still capture well the multipath channel effect using the RAKE receiver, which also provides diversity gain, as each multipath component carries information along with it.

No matter whether we start sampling at a time delay of zero seconds from the line-of-sight component of Bob's packet (i.e., chip-level synchronized) or $\tau-\Delta$ seconds $\left((\tau-\Delta)<T_{c}\right)$ (i.e., asynchronously), this will not affect the results by much, since each multipath component (as we later show in the experimental section in Fig. 12) does not necessarily arrive exactly at every $T_{c}$ time interval. However, we can still capture the channel multipath adequately. In other words, even though the receiver may fail to capture the first multipath component of Bob's signal it can still capture other components using the RAKE filter.

In case of only a few dominant multipath arrivals as long as the multipath arrivals are random and a number of them can be captured using the RAKE receiver our scheme will still work. In case of either no multipath (i.e., a single line-of-sight communication channel) or a multipath fading channel in which the multipath components are located at exactly every chip interval $T_{c}$ then chip-level synchronization is essential for our scheme to work. However, as we mentioned before, underwater acoustic channel often times experiences strong multipath fading and in addition to that the locations of multipath components are random [28]. Therefore, it is very unlikely (in practice impossible) that all the components will be located at every other chip interval i.e., at $T_{c}, 2 T_{c}, 3 T_{c}$, etc. Accordingly, using the proposed method we can still extract the information of interest.

\footnotetext{
${ }^{2}$ To determine which packet is leading/lagging, each user will decode the packet header/trailer that includes transmit and receive user IDs, more details on the packet header and trailer is discussed in [1].
} 


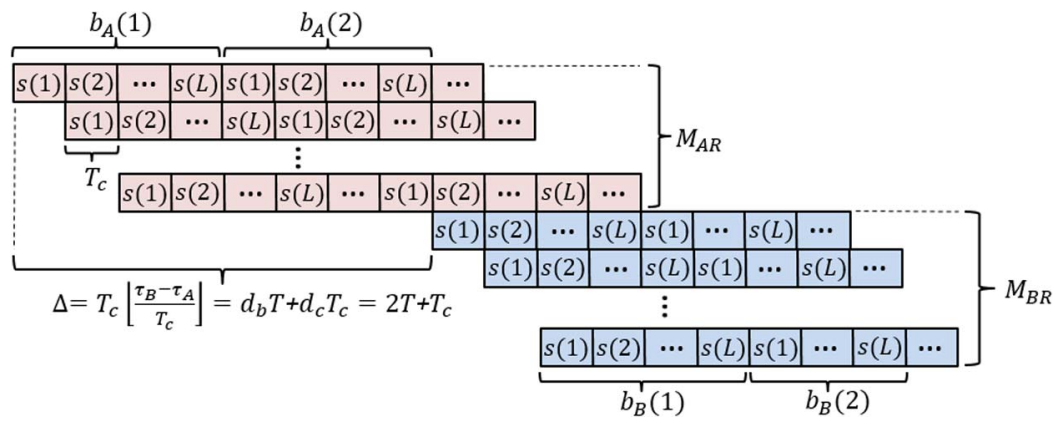

Fig. 3. Alice's and Bob's packets overlapped at the relay asynchronously.

In this exposition, we also assume the number of resolvable multipaths at the relay $M_{A R}=M_{B R}+1=M$, which can be generalized to different number of multipaths. Bob's information/pilot bit index relative to Alice's can be expressed as $i_{B}=$ $i-d_{b}$. The received signal at the relay, (6), will have $\mathbf{S}_{B}\left(i_{B}\right)$ in place of $\mathbf{S}_{B}(i)$ to account for the asynchronous packet arrival. Thus, the equations (10)-(13) for Bob's case can be rewritten as (16)-(19), shown at the bottom of the page.

The matrices (17)-(19) can be constructed knowing the relative bit and chip delay of the two packets, which is estimated using the chirp signal, as discussed earlier. Hence, the matrices (17)-(19) differ from (11)-(13) by having the rows shifted up by the number of chip delays, $d_{c}$. In addition to that, an all zero column vector is appended to the right of the matrices (11)-(13) to account for the difference in tap delay line, i.e., $M_{A R}-M_{B R}=1$. In general, $M_{A R}-M_{B R}$ number of zero vectors will need to be appended. In (17)-(19), $s(l)=0$ for $l \notin\{1,2, \ldots, L\}$.

The two overlapped packets received at the relay are shown in Fig. 3. Alice's packet, followed by $M_{A R}$ multipath components, arrives before Bob's packet. In this example we assume the relative time delay $\Delta=2 T+T_{c}$.

$$
\mathbf{S}_{B}\left(i_{B}\right)=\mathbf{S}_{B}^{0}\left(i_{B}\right)+\mathbf{S}_{B}^{+}\left(i_{B}\right)+\mathbf{S}_{B}^{-}\left(i_{B}\right),
$$

where

$$
\mathbf{S}_{B}^{0}\left(i_{B}\right)=b_{B}\left(i_{B}\right)\left[\begin{array}{cccccc}
s\left(d_{c}+1\right) & s\left(d_{c}\right) & s\left(d_{c}-1\right) & \cdots & 0 & 0 \\
\vdots & s\left(d_{c}+1\right) & s\left(d_{c}\right) & \ddots & \vdots & \vdots \\
s(L) & \vdots & s\left(d_{c}+1\right) & \ddots & 0 & 0 \\
0 & s(L) & \vdots & & s(1) & 0 \\
\vdots & \vdots & s(L) & & \vdots & \vdots \\
0 & 0 & 0 & \ddots & s(L) & 0 \\
0 & 0 & 0 & \cdots & 0 & 0
\end{array}\right]_{(L+M-1) \times M}
$$

$$
\begin{aligned}
& \mathbf{S}_{B}^{+}\left(i_{B}\right)=b_{B}\left(i_{B}+1\right)\left[\begin{array}{cccc}
0 & \cdots & 0 & 0 \\
\vdots & \ddots & \vdots & \vdots \\
0 & & 0 & 0 \\
s(1) & \ddots & \vdots & 0 \\
\vdots & \ddots & 0 & \vdots \\
s\left(M_{B R}+d_{c}-1\right) & \cdots & s(1) & 0
\end{array}\right]_{(L+M-1) \times M} \\
& \mathbf{S}_{B}^{-}\left(i_{B}\right)=b_{B}\left(i_{B}-1\right)\left[\begin{array}{cccccc}
0 & s\left(L+d_{c}\right) & s\left(L+d_{c}-1\right) & \ldots & s\left(L-M_{B R}+d_{c}+1\right) & 0 \\
0 & 0 & s\left(L+d_{c}\right) & \ldots & s\left(L-M_{B R}+d_{c}+2\right) & 0 \\
\vdots & \vdots & 0 & \ddots & \vdots & \vdots \\
0 & 0 & 0 & & s(L) & 0 \\
\vdots & \vdots & \vdots & \ddots & \vdots & \vdots \\
0 & 0 & 0 & \cdots & 0 & 0
\end{array}\right]_{(L+M-1) \times M}
\end{aligned}
$$




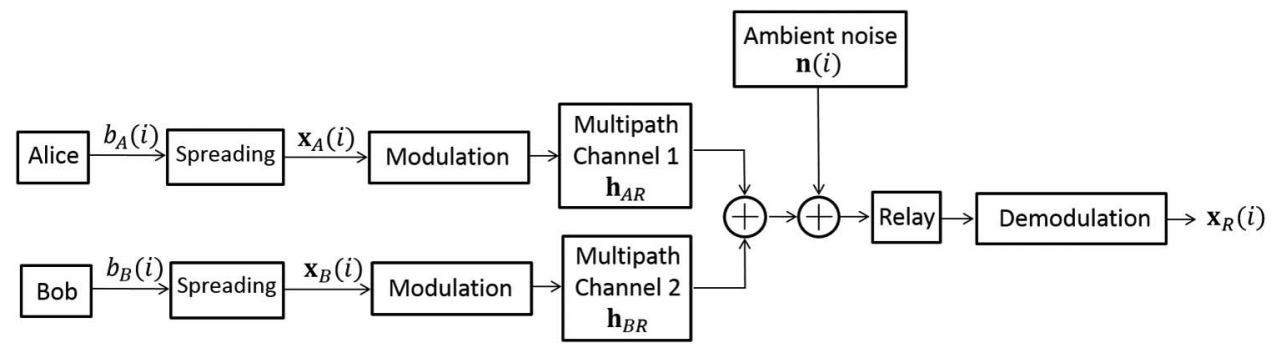

Fig. 4. System model for ANC-CDMA.

The ANC-CDMA system model is shown in Fig. 4.

3) Joint Channel Estimation: We now show how to estimate the CSIs from Alice-to-relay and Bob-to-relay jointly. We first address the synchronous transmission case and latter generalized to the asynchronous case. Let us define

$$
\mathbf{S}_{A B}(i) \triangleq\left[\sqrt{E_{A}} \mathbf{S}_{A}(i), \sqrt{E_{B}} \mathbf{S}_{B}(i)\right]_{(L+M-1) \times 2 M},
$$

and

$$
\mathbf{h}_{A B} \triangleq\left[\begin{array}{l}
\mathbf{h}_{A R} \\
\mathbf{h}_{B R}
\end{array}\right]_{2 M \times 1} .
$$

Using (20) and (21) we may rewrite (6) in a more compact form as

$$
\mathbf{r}_{R}(i)=\mathbf{S}_{A B}(i) \mathbf{h}_{A B}+\mathbf{n}(i), \quad i=1,2, \ldots
$$

Before jointly estimating the channel coefficients, $\mathbf{h}_{A R}$ and $\mathbf{h}_{B R}$, we first define the pseudo-inverse of $\mathbf{S}_{A B}(i)$ for $(L+M-$ 1) $>2 M$ using the Moore-Penrose pseudo-inverse formula as [29]

$$
\mathbf{S}_{A B}^{\dagger}(i) \triangleq\left[\mathbf{S}_{A B}(i)^{H} \mathbf{S}_{A B}(i)\right]^{-1} \mathbf{S}_{A B}(i)^{H} .
$$

We can obtain the least square (LS) estimate of $\mathbf{h}_{A B}$ by minimizing the following squared error quantity

$$
\tilde{\mathbf{h}}_{A B}=\arg \min _{\boldsymbol{h}_{A B}}\left\|\mathbf{r}_{R}(i)-\mathbf{S}_{A B}(i) \mathbf{h}_{A B}\right\|^{2} .
$$

The marginal solution of (24) can be estimated by sample averaging over a data record of $N_{p}$ pilot bits as

$$
\hat{\mathbf{h}}_{\mathbf{A B}}=\frac{1}{N_{p}} \sum_{i=1}^{N_{p}} \mathbf{S}_{A B}^{\dagger}(i) \mathbf{r}_{R}(i) .
$$

We can obtain an accurate estimate of $\mathbf{h}_{A B}$ if and only if (20) is of full rank. This condition is satisfied if (20) contains $2 M$ independent vectors. In this work, we use columns of a Sylvester-Hadamard matrix, $\mathbf{H}_{L}$ with elements +1 or -1 , of order $L=2^{n}, n=2,3, \ldots$, as our spreading codes. The Sylvester-Hadamard matrix has good autocorrelation and crosscorrelation properties [30], [31]. Rows (and columns) of the $\mathbf{H}_{L}$ are mutually orthogonal to each other. For a spreading code of order $L=4$ extracted from $\mathbf{H}_{L}$, the above condition cannot be satisfied for $M=3$, hence a spreading code length of $L=8$ or longer needs to be used in this case.

In practice, Alice and Bob initially will utilize a long spreading code, e.g., $L=128$, and adaptively reduce its length depending on the channel conditions. The long spreading code will help to satisfy the dimensionality requirement $(L+M-$ $1>2 M$ ) for the first packet transmission. For subsequent packet transmissions, the relay will estimate the multipath delay spread of the two channels (i.e., $M_{A R}$ and $M_{B R}$ ) by chirpmatched-filtering the received signals, discussed in greater detail in Section V-B3, and accordingly select a spreading code of minimum length that satisfies the dimensionality requirement.

The noisy nature of the acoustic channel results in SNR degradation in joint channel estimation, an approximation of the SNR degradation is given in [32] as

$$
d_{c e} / d B=10 \cdot \log _{10}\left[1+\operatorname{tr}\left\{\left[\mathbf{S}_{A B}(i)^{H} \mathbf{S}_{A B}(i)\right]^{-1}\right\}\right] .
$$

Therefore, it is important to select the training sequences for both nodes with very low cross-correlation properties to minimize the noise enhancement, $\mathbf{S}_{A B}^{\dagger}(i) \mathbf{n}(i)$, which can be shown to be equal to $\operatorname{tr}\left\{\left[\mathbf{S}_{A B}(i)^{H} \mathbf{S}_{A B}(i)\right]^{-1}\right\}$. We can observe from (26) that the optimal set of training sequences must satisfy $\mathbf{S}_{A B}(i)^{H} \mathbf{S}_{A B}(i)=\mathcal{P} \mathbf{I}_{2 M}$, where $\mathcal{P}$ is a given constant value. Accordingly, to minimize the noise enhancement we utilize two orthogonal sequences of pilot bits extracted again from columns of $\mathbf{H}_{L}$, of order $L=2^{n}, n=4,5, \ldots$

The CSIs from Alice-to-relay and Bob-to-relay are computed as follows

$$
\begin{array}{r}
\hat{\mathbf{h}}_{A R}=\left[\begin{array}{ll}
\mathbf{I}_{M} & \mathbf{0}_{M}
\end{array}\right] \hat{\mathbf{h}}_{A B}, \\
\hat{\mathbf{h}}_{B R}=\left[\begin{array}{ll}
\mathbf{0}_{M} & \mathbf{I}_{M}
\end{array}\right] \hat{\mathbf{h}}_{A B} .
\end{array}
$$

The mean square error (MSE) per real channel coefficient $\mathbf{h}_{A R}$, is given by

$$
M S E=\mathbb{E}\left\{\left\|\mathbf{h}_{A R}-\hat{\mathbf{h}}_{A R}\right\|^{2}\right\} .
$$

We evaluate the MSE of channel estimates $\hat{\mathbf{h}}_{A R}$ and $\hat{\mathbf{h}}_{B R}$ for various SNR values and two different number of pilot bits $N_{p}$ in Section V-A1.

For the case of asynchronous packet arrival, we apply the joint channel estimation discussed above, replacing $\mathbf{S}_{B}(i)$ with $\mathbf{S}_{B}\left(i_{B}\right)$ in (20). Note that in the above example, shown in Fig. 3, the non-overlapped portion of the bits, i.e., $b_{A}(1)$ and $b_{A}(2)$, the $\mathbf{S}_{B}\left(i_{B}\right)$ in (20) is an all zero matrix.

4) Receiver Design: For the synchronous packet arrival case, to decode the information bits, we use the estimated CSIs, $\hat{\mathbf{h}}_{A R}, \hat{\mathbf{h}}_{B R}$, and design an adaptive RAKE-matched-filter that decides on the transmitted bit of the user of interest (Alice) 
based on the sum of the individual $M$ path-correlator outputs, which can be equivalently characterized by the normalized static $(L+M-1)$-tap FIR filter given by

$$
\mathbf{w}_{R A K E_{M F}}=\frac{\mathbf{S}_{M F} \hat{\mathbf{h}}_{A R}}{\left(\mathbf{S}_{M F} \hat{\mathbf{h}}_{A R}\right)^{H}\left(\mathbf{S}_{M F} \hat{\mathbf{h}}_{A R}\right)},
$$

where

$$
\mathbf{S}_{M F}=\left[\begin{array}{cccc}
s(1) & 0 & \cdots & 0 \\
\vdots & s(1) & & \vdots \\
s(L) & \vdots & \ddots & 0 \\
0 & s(L) & & s(1) \\
\vdots & \vdots & \ddots & \vdots \\
0 & 0 & \cdots & s(L)
\end{array}\right]_{(L+M-1) \times M}
$$

represents the $M$ path-correlator outputs, which can be constructed knowing the number of multipaths, $M$.

Before decoding the information bits, we first cancel the inter-symbol-interference (ISI) resulting from the previously decoded bits. Moreover, we cancel the estimated interfered data bits from Bob and, employing the adaptive RAKEmatched-filter proposed in (30), the information bits $\left(b_{A}(j), j=\right.$ $1,2, \ldots$ ) of the user of interest (Alice) are decoded as follows

$\hat{b}_{A}(j)=\operatorname{sgn}\left(\mathfrak{R e}\left[\mathbf{w}_{R A K E_{M F}^{H}}\left(\hat{\mathbf{r}}_{R}(j)-\mathbf{S}_{A}^{-}(j) \hat{\mathbf{h}}_{A R}-\mathbf{S}_{B}(j) \hat{\mathbf{h}}_{B R}\right)\right]\right)$,

where $\mathbf{S}_{A}^{-}(j) \hat{\mathbf{h}}_{A R}$ is the ISI of the previously decoded bit of Alice and $\mathbf{S}_{B}(j) \hat{\mathbf{h}}_{B R}$ is the estimate of the interfered data bit originally transmitted by Bob.

Similarly, for the asynchronous packet arrival case, to decode the information bits we apply (32) by first replacing $\mathbf{S}_{B}(j)$ with the delayed version, i.e., $\mathbf{S}_{B}\left(j_{B}\right)$, where $j_{B}=j-b_{d}$.

\section{B. Bi-Directional CDMA-Based ANC}

We now consider a CDMA-based bi-directional network shown in Fig. 2(b) in which Alice and Bob would like to exchange information to one another using the same spreading code previously assigned to them.

1) Transmission Phase I: Alice and Bob concurrently transmit their packets $P_{A}$ and $P_{B}$ to the relay. The baseband supervised and information bits transmitted by Alice and Bob are given by (2) and (3), respectively.

After the relay receives the two packets $P_{A}$ and $P_{B}$ from Alice and Bob, respectively, which interfere with each other as expressed by (6), it first performs carrier demodulation and appends a preamble at the beginning of the packet, which contains three identical chirp signals each separated by a guardband and information on the multipath delay spread of the two channels (i.e., $M_{A R}$ and $M_{B R}$ ) estimated from the preambles of the received signal, discussed in Section IV-A and Section V-B, to inform Alice and Bob on the length of code to select to satisfy the dimensionality requirement $(L+M-1>2 M)$ for the subsequent packet transmission. In addition, it inserts a new set of $N_{p}$ pilot bits before each static block duration $\left(T_{C T}\right)$ within the packet. The pilot bits, spread with the same spreading sequence (4), will be used by Alice and Bob to estimate and compensate for the channel effects introduced in the transmission phase II. Inserting the new set of pilot bits at the relay, instead of at each user side, will simplify estimation and equalization of the channel effects introduced during the transmission of the overlapped packet by the relay to both Alice and Bob. In other words, we avoid the extra supervised bits inserted at the relay to undergo an additional multipath Rayleigh fading channel (i.e., Alice-to-relay and Bob-to-relay) that would have experienced if instead they were added at each node in the first place. The relay amplifies the new packet $P_{R}$ and forwards it to Alice and Bob in the transmission phase II.

We now discuss how Bob can successfully decode Alice's packet having received $P_{R}$ from the relay. Similar analysis can be carried out on Alice's receiver side to decode Bob's packet.

2) Transmission Phase II: After carrier demodulation, chipmatched-filtering and sampling at the chip rate over multipath extended bit period, the received signal by Bob is comprised of the pilot bits introduced by the relay along with the superimposed pilot and information bits transmitted by Alice and Bob. The $i^{\text {th }}$ pilot bit of $P_{R}$ received by Bob is denoted by

$$
\mathbf{r}_{B}(i)=\sqrt{E_{R}} \mathbf{S}_{R}(i) \mathbf{h}_{R B}+\mathbf{n}_{R B}(i), \quad i=1,2, \ldots,
$$

where

$$
\mathbf{h}_{R B}=\left[h_{R B}(1), h_{R B}(2), \ldots, h_{R B}\left(M_{R B}\right)\right]^{H}
$$

is the relay-to-Bob multipath channel coefficients. $h_{R B}(q)$ represents the $q^{\text {th }}$ resolvable path coefficients modeled as complex Gaussian random variables (Rayleigh-distributed) that remain constant during $T_{C T}$ block length (i.e., quasi-static fading), $E_{R}$ denotes the transmit energy per bit, $\mathbf{n}_{R B}$ is the acoustic noise in the underwater communication channel with the power spectral density (15), and

$$
\mathbf{S}_{R}(i)=\mathbf{S}_{R}^{0}(i)+\mathbf{S}_{R}^{+}(i)+\mathbf{S}_{R}^{-}(i) .
$$

The matrices $\mathbf{S}_{R}^{0}(i), \mathbf{S}_{R}^{+}(i)$, and $\mathbf{S}_{R}^{-}(i) \in \mathbb{R}^{\left(L+M_{R B}-1\right) \times M_{R B}}$ are denoted by (11), (12) and (13) with $k \in\{R\}$, which correspond to the spreading code matrices generated due to transmission of pilot bits $b_{R}(i), b_{R}(i+1)$, and $b_{R}(i-1)$, respectively, by the relay.

3) MMSE Equalizer: Before applying channel equalization we first estimate the channel coefficients $\mathbf{h}_{R B}$, introduced during the transmission from relay-to-Bob, by using the MMSE estimator and sample averaging over a data record of $N_{p}$ pilot bits as

$$
\hat{\mathbf{h}}_{R B}=\frac{1}{N_{p}} \sum_{i=1}^{N_{p}} \mathbf{S}_{R}^{\dagger}(i) \mathbf{r}_{B}(i),
$$

where

$$
\mathbf{S}_{R}^{\dagger}(i) \triangleq\left[\mathbf{S}_{R}(i)^{H} \mathbf{S}_{R}(i)\right]^{-1} \mathbf{S}_{R}(i)^{H}
$$

is the pseudo-inverse of $\mathbf{S}_{R}(i)$ for $\left(L+M_{R B}-1\right)>M_{R B}$.

We rewrite (33) as

$$
\mathbf{r}_{B}(i)=\sqrt{E_{R}} \mathbf{H}_{R B} \mathbf{x}_{R}(i)+\mathbf{n}_{R B}(i), \quad i=1,2, \ldots,
$$


where

$$
\mathbf{x}_{R}(i)=b_{R}(i) \sqrt{E_{R}} \mathbf{s},
$$

is the $i^{\text {th }}$ direct sequence spread pilot bit transmitted by the relay and $\mathbf{H}_{R B} \in \mathbb{C}^{\left(L+M_{R B}-1\right) \times L}$, defined as

$$
\mathbf{H}_{R B} \triangleq\left[\begin{array}{cccc}
h_{R B}(1) & 0 & \cdots & 0 \\
\vdots & h_{R B}(1) & & \vdots \\
h_{R B}\left(M_{R B}\right) & \vdots & \ddots & 0 \\
0 & h_{R B}\left(M_{R B}\right) & & h_{R B}(1) \\
\vdots & \vdots & \ddots & \vdots \\
0 & 0 & \ldots & h_{R B}\left(M_{R B}\right)
\end{array}\right]
$$

corresponds to the Toeplitz channel covariance matrix from relay-to-Bob.

Using $\hat{\mathbf{h}}_{R B}$, we design the MMSE equalizer as

$$
\mathbf{H}_{R B}^{\dagger} \triangleq\left(\hat{\mathbf{H}}_{R B}^{H} \hat{\mathbf{H}}_{R B}+\sigma_{R B}^{2} \mathbf{I}_{L}\right)^{-1} \hat{\mathbf{H}}_{R B}^{H},
$$

where $\sigma_{R B}^{2} \mathbf{I}_{L}$ is the noise covariance matrix, which can be estimated during the node's idle period.

Bob will use (41) to equalize the received signal as follows

$$
\tilde{\mathbf{r}}_{R}(i)=\mathbf{H}_{R B}^{\dagger} \mathbf{r}_{B}(i), \quad i=1,2, \ldots
$$

After removing the pilot bits introduced by the relay from (42), Bob reconstructs an estimate of the packet (6) that was originally received by the relay node in phase I, which can be expressed as

$$
\hat{\mathbf{r}}_{R}(i)=\mathbf{S}_{A B}(i) \mathbf{h}_{A B}+\mathbf{n}(i)+\mathbf{e}(i), \quad i=1,2, \ldots,
$$

where $\mathbf{e}(i)=\hat{\mathbf{r}}_{R}(i)-\mathbf{r}_{R}(i)$ denotes the error introduced during channel equalization.

To decode Alice's packet, Bob will jointly estimate the two CSIs, $\hat{\mathbf{h}}_{A R}$ and $\hat{\mathbf{h}}_{B R}$, using (27) and (28), respectively, and use the receiver proposed in Section IV-A4. Note that Bob does not need to equalize the channel effects introduced in phase I transmission as the receiver designed in (30) already takes that into consideration.

\section{Performance Evaluation}

In this section, using simulations and real testbed experiments, we evaluate the performance of unidirectional and bidirectional ANC-CDMA schemes and compare them with the conventional multi-user (MU) DS-CDMA in terms of average BER. The average MSE of the joint channel estimation is also studied.

\section{A. Simulation Results}

Simulations of the proposed ANC-CDMA were performed in Matlab using the unidirectional and bi-directional network topologies discussed in Sections IV-A and IV-B, respectively.

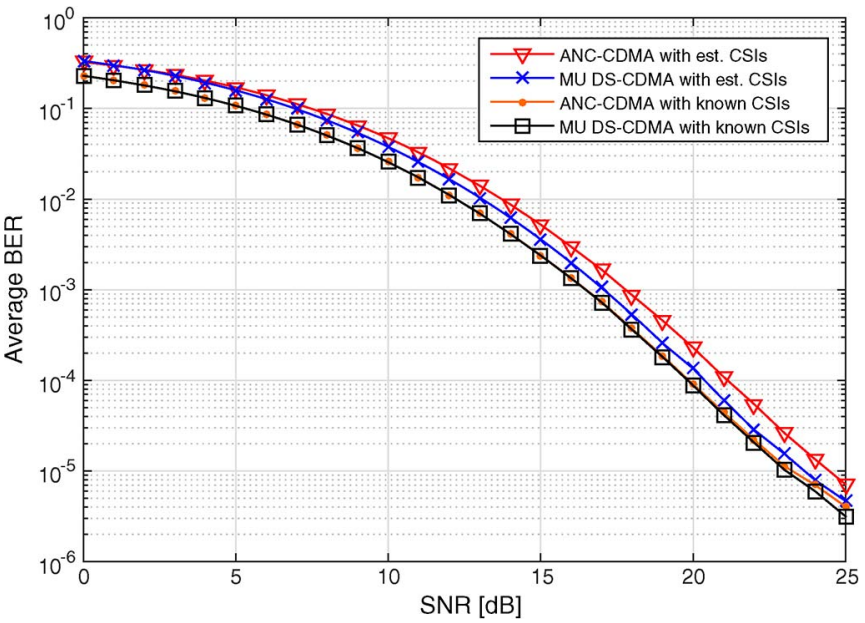

Fig. 5. Average bit error rate (BER) versus signal-to-noise ratio (SNR). ( $L=$ $32, N_{P}=16$, fragment size $=125$ Bytes, payload size $=1.25 \mathrm{kBytes}$ )

1) Unidirectional ANC: We first evaluate the performance of unidirectional ANC-CDMA, in which both nodes concurrently transmit their packets, which collide at the relay node. Both channels, Alice-to-relay and Bob-to-relay, are modeled as Rayleigh fading in which the multipath channel coefficients are considered as discrete independent zero-mean complex Gaussian random variables of variance one, and the number of multipaths are randomly selected from the range of values 1-15 for each tap delay line. The relay node estimates the CSIs from the two nodes and cancels the interference, Bob's packet, before decoding the information of interest, Alice's packet. Similar procedure is performed to simulate the multi-user DSCDMA scheme, in which the interfered packet, known a priori, is suppressed before decoding the packet of interest. The DSCDMA chip waveforms were selected from the columns of a Sylvester-Hadamard matrix of order $L=32$. For the case of ANC-CDMA, the $10^{\text {th }}$ column was selected while for the case of multi-user DS-CDMA, $10^{\text {th }}$ and $26^{\text {th }}$ columns were selected. The transmitted packets are divided into fragments and the pilot bits are inserted before each fragment. The fragment size is determined based on the average coherence time of a shallow water acoustic channel, which is in the order of a few seconds for a transmission frequency of $10 \mathrm{kHz}$ [33]. Accordingly, a fragment size of 125 Bytes is selected. Unless otherwise stated, the parameters used for simulations are payload size $=$ $1.25 \mathrm{kBytes}$, fragment size $=125$ Bytes, number of pilot bits $N_{P}=16$, spreading code length $L=32$, shipping noise $s=0.1$ (i.e., light shipping activity), wind speed $w=8 \mathrm{~m} / \mathrm{s}$, and the transmit frequency $f_{c}=11.5 \mathrm{kHz}$, which corresponds to the carrier frequency of acoustic modems used in the actual experiments. The probability of error conditioned on channel coefficients is averaged over 1000 independent channel realizations.

In Fig. 5 we plot the average BER for various SNR values. Since MU DS-CDMA utilizes orthogonal spreading codes, it is less sensitive to channel estimation errors, and thus its performance is very close to MU DS-CDMA with known CSIs. We can also observe that the performance of unidirectional ANC-CDMA is quite close to the conventional MU DS-CDMA scheme. When perfect knowledge of CSIs is available, the performance of ANC-CDMA will be similar to MU DS-CDMA with known 


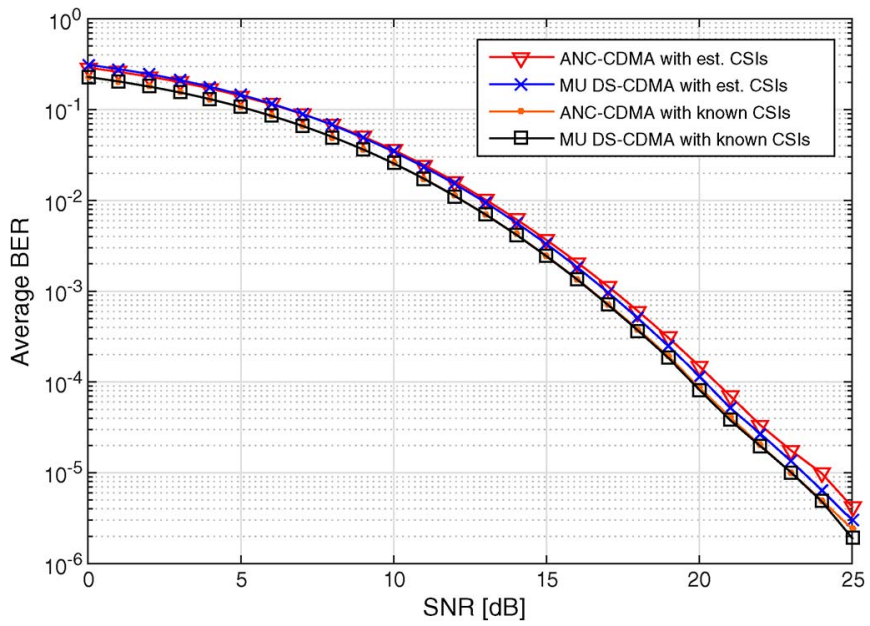

Fig. 6. Average BER versus SNR. $\left(L=32, N_{P}=32\right.$, fragment size $=$ 125 Bytes, payload size $=1.25$ kBytes).

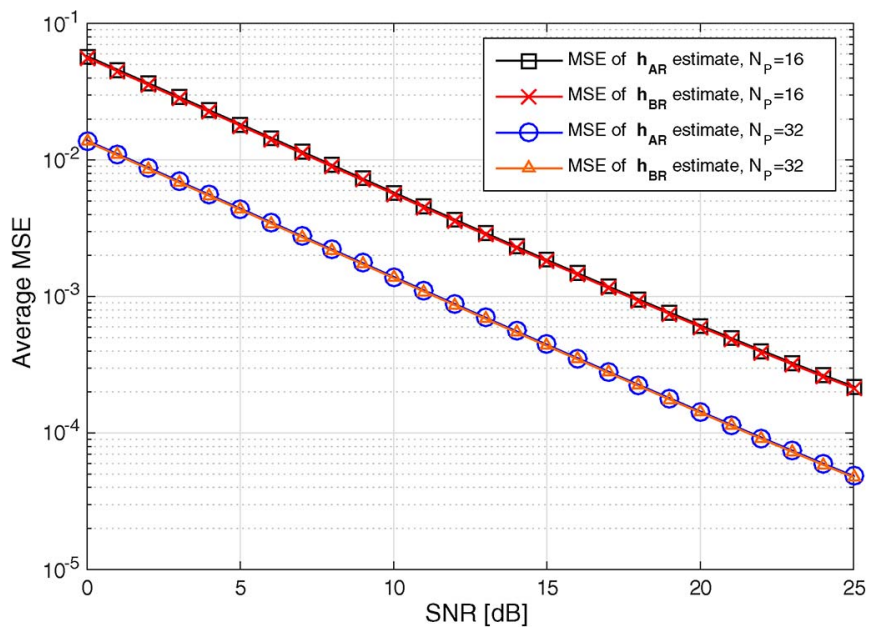

Fig. 7. Average MSE of CSI estimate versus SNR. $\left(L=32, N_{P}=16\right.$ and $N_{P}=32$, fragment size $=125$ Bytes and payload size $=1.25$ kBytes $)$.

CSIs, as the interfering packet can completely be removed. To achieve a BER of $10^{-4}$, an SNR of $20 \mathrm{~dB}$ and $21 \mathrm{~dB}$ are required with the conventional MU DS-CDMA and ANC-CDMA schemes, respectively. For a penalty of $1 \mathrm{~dB}$ the proposed scheme can improve the channel utilization by up to $50 \%$.

In Fig. 6 we plot the average BER for various SNR values respectively for $N_{P}=32$ (instead of $N_{P}=16$ ). We observe that the performance of the unidirectional ANC-CDMA improves at the cost of an increase in the number of pilot bits. To achieve a BER of $10^{-4}$, the ANC-CDMA sacrifices less than $1 \mathrm{~dB}$ in SNR for an increase in channel utilization by up to $50 \%$.

In Fig. 7, the performance of channel estimation, measured as the MSE per real channel coefficient, is shown as a function of SNR. We observe that by increasing the number of pilot bits from $N_{P}=16$ to $N_{P}=32$ the CSI estimation improves significantly. Moreover, as the SNR at the receiver increases, the MSE approaches to zero.

2) Bi-Directional ANC: Similarly, simulations of bidirectional ANC-CDMA were carried out following the discussions in Section IV-B.

In Fig. 8 we plot the average BER for various SNR values. As we can see, the performance of ANC-CDMA is slightly worse

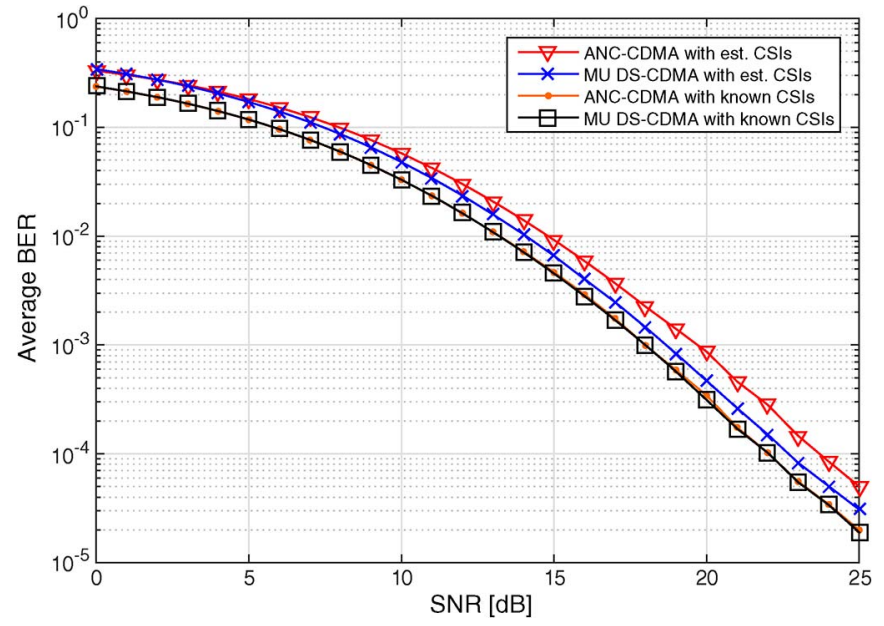

Fig. 8. Average BER versus SNR. $\left(L=32, N_{P}=16\right.$, fragment size $=$ 125 Bytes, payload size $=1.25$ kBytes).

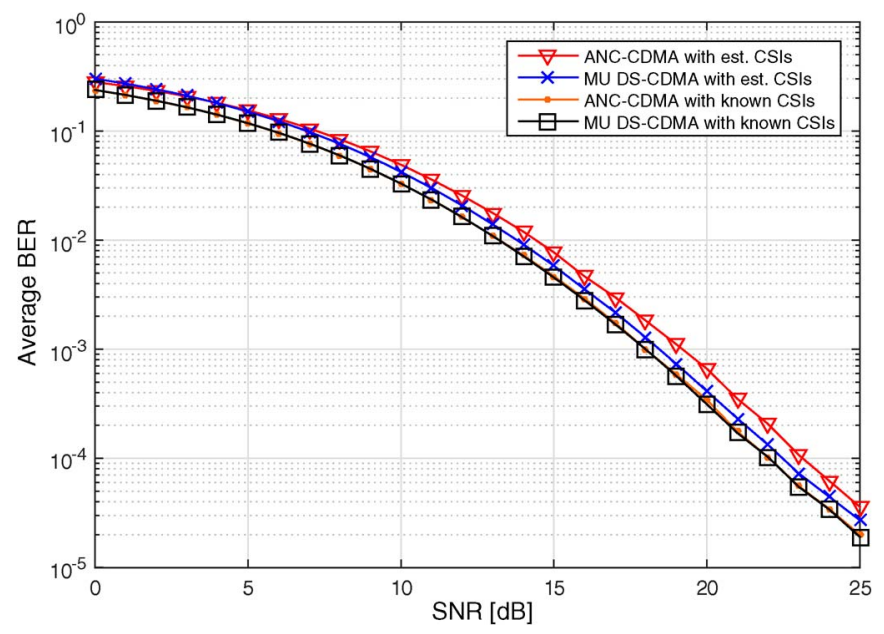

Fig. 9. Average BER versus SNR. $\left(L=32, N_{P}=32\right.$, fragment size $=$ 125 Bytes, payload size $=1.25 \mathrm{kBytes})$.

than unidirectional case, due to the extra channel equalization requirement, but still it is close to the conventional MU DSCDMA scheme. To achieve a BER of $10^{-4}$, an SNR of $22 \mathrm{~dB}$ and $23.54 \mathrm{~dB}$ is required with the conventional MU DS-CDMA and ANC-CDMA schemes, respectively. For a penalty of $1.54 \mathrm{~dB}$ the proposed scheme can improve the channel utilization by up to $100 \%$.

In Fig. 9 we plot the average BER for various SNR values for $N_{P}=32$ (instead of $N_{P}=16$ ). We observe that the performance of ANC-CDMA improves at the cost of an increase in the number of pilot bits. To achieve a BER of $10^{-4}$, the ANCCDMA sacrifices about $1 \mathrm{~dB}$ in SNR for an increase in channel utilization by up to $100 \%$.

\section{B. Experimental Evaluation}

In this section we present the experimental results to demonstrate the performance of the proposed ANC-CDMA scheme. Telesonar SM-975 SMART modems designed by Teledyne Benthos were used as transmitter and receiver during the experiments.

The Telesonar SM-975 acoustic modem is designed for deep and shallow water communication. The acoustic data 


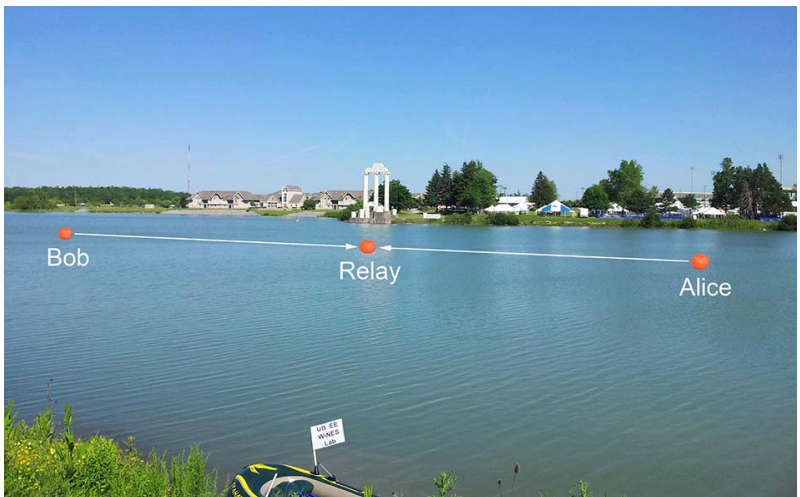

Fig. 10. Actual deployment of the three underwater acoustic modems in Lake LaSalle at the University at Buffalo.

modulation methods supported by the modem are multiple frequency-shift keying (MFSK) and phase-shift keying (PSK), as well as playing and recording an arbitrary waveform defined through standard Matlab primitives. Later option was used in our experiments to generate and transmit DS-CDMA modulated data streams.

1) Transmitter Modem Operation: The modem uses an omnidirectional transducer that operates in the 9-14 kHz (LF) low frequency band. The waveform-play feature of the modem enables transmissions of baseband complex data with a bandwidth of $5,120 \mathrm{~Hz}$ sampled at $10,240 \mathrm{~Hz}$. The data packets were generated offline, converted into a audio file in 16-bit format, and uploaded on the modems through the RS-232 serial interface. The DS-CDMA chip waveforms were selected from the columns of a Sylvester-Hadamard matrix of order $L=32$. For the case of ANC-CDMA, the $10^{\text {th }}$ column was selected while for the case of multi-user DS-CDMA, $10^{\text {th }}$ and $26^{\text {th }}$ columns were selected. Pulse shaping was done using squareroot raised-cosine with roll-off factor $\beta=0.5$ and a chip rate of $R_{c}=2,048$ chips/s was generated.

2) Receiver Modem Operation: The modem, acting as a receiver, performs recording of the transmitted signals as baseband complex data. Data packets are recorded in the secure digital high capacity (SDHC) card at $10,240 \mathrm{~Hz}$ sampling rate as stereo waveform audio files in 16-bit format with the right channel as the imaginary part and the left channel as the real part. The recording of the data packets were done by sending commands through the RS-232 serial interface from the host processor.

Experiments of unidirectional and bi-directional ANCCDMA were conducted in Lake LaSalle at the University at Buffalo using three Telesonar SM-975 SMART modems. The actual deployment of the underwater acoustic modems is shown in Fig. 10, with three orange buoys floating on the surface of the lake, each attached to the Telesonar SM-975 acoustic modem, weighing $18 \mathrm{~kg}$, and submerged using an anchor.

The three modems were deployed at a depth of $1 \mathrm{~m}$ above the lake floor in the locations shown in Fig. 10. The distances Aliceto-relay and Bob-to-relay were set to approximately $d_{A R}=$ $110 \mathrm{~m}$ and $d_{B R}=110 \mathrm{~m}$, respectively. A total of $1.25 \mathrm{kByte}$ of data was transmitted and each experiment was repeated 20 times with different transmit power levels. The transmit

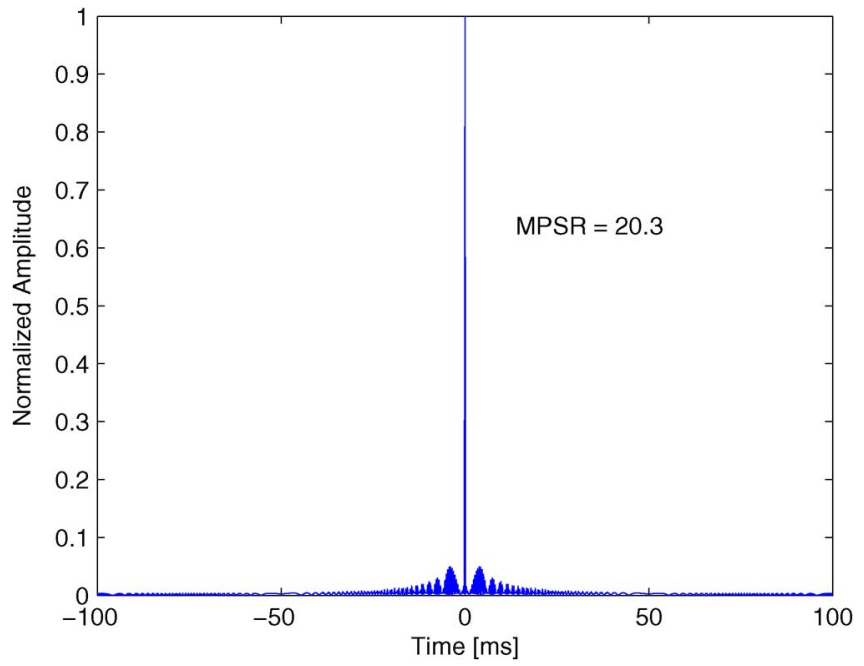

Fig. 11. Autocorrelation of the transmitted chirp signal.

power levels provided by the SM-975 modem are in the range from $-10.5 \mathrm{~dB}(1.78 \mathrm{~W})$ to $0 \mathrm{~dB}(20 \mathrm{~W})$, with $1.5 \mathrm{~dB}$ increments.

3) Unidirectional ANC: Initially, Alice was selected to transmit packet $P_{A}$ to the relay node. In the next round nodes Alice and Bob simultaneously transmit packets $P_{A}$ and $P_{B}$ respectively to the intermediate relay node using the same power level. A laptop, on an inflatable boat, was used to coordinate the transmissions of the packets through a serial port interface. All the acoustic modems are equipped with a data recorder that has a storage capacity of 64 GBytes. The experiments were conducted for different transmit power levels. The raw data were extracted and analyzed in Matlab and the average values are presented in the plot.

An ideal channel impulse response (i.e., no multipath case), obtained by auto-correlating the preamble/chirp signal, is shown in Fig. 11. We can see that the chirp signal has ideal characteristics, a sharp main lobe with extremely low sidelobes with mainlobe to peak sidelobe ratio $M P S R=20.3$.

Fig. 12 shows a snapshot of the channel impulse responses (CIRs) for Alice-to-relay and Bob-to-relay channels, obtained by chirp matched filtering the received acoustic signal at the relay, respectively.

We observe that the channel is prone to high multipath effects with a multipath delay spread of about $6.2 \mathrm{~ms}$ and $7.1 \mathrm{~ms}$ for Alice-to-relay and Bob-to-relay, respectively. We use energy thresholding in estimating the multipath delay spread. The normalized multipath components magnitude below 0.2 are considered as interference and/or noise, and thus are ignored. From the multipath delay spread the relay can compute $M_{A R}$ and $M_{B R}$, the length of tap-delay line needed for channel estimation and the receiver design, in this example they are 13 and 14, respectively. In practice, a few taps longer were selected to be on the safe side. The observed strong multipath effect is due to the very shallow depth of the lake, only about $4.5 \mathrm{~m}$.

4) Bidirectional ANC: Similarly, experiments of bidirectional ANC-CDMA were carried out following the discussions in Section IV-B. In phase I, Alice and Bob simultaneously transmit packets $P_{A}$ and $P_{B}$ respectively to the intermediate relay node. The relay first carrier demodulates, 


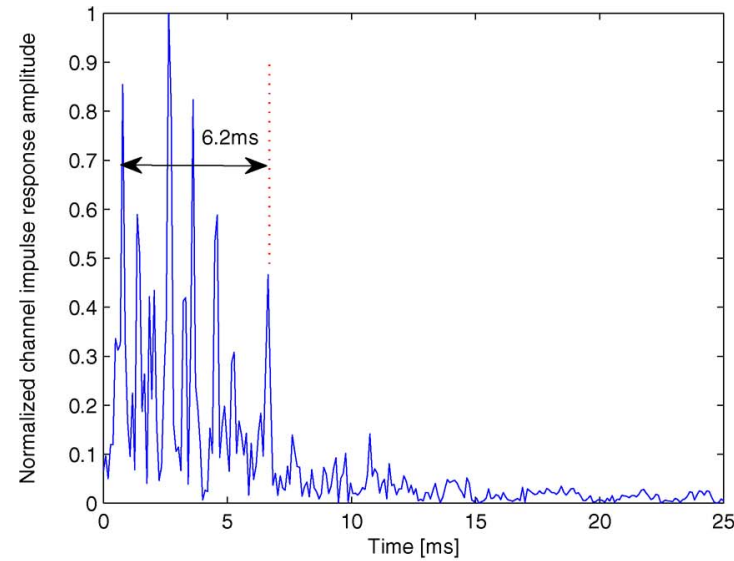

(a)

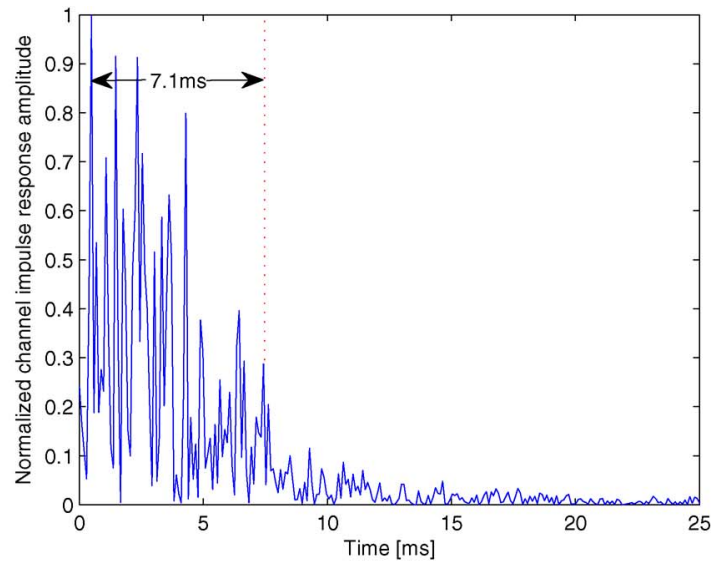

(b)

Fig. 12. Normalized channel impulse response: (a) Alice-to-relay, (b) Bob-to-relay.

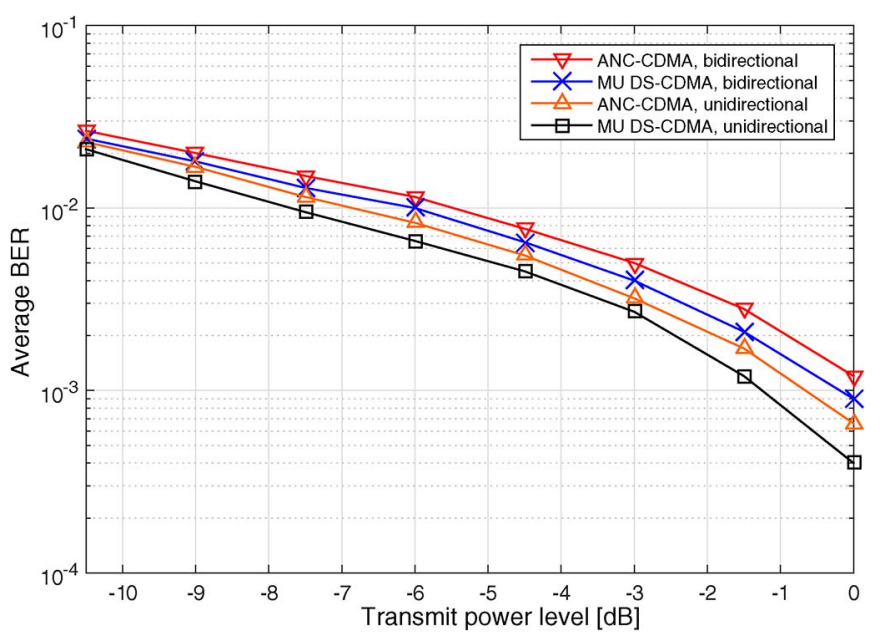

Fig. 13. Average BER versus transmit power level. $\left(L=32, N_{P}=32\right.$, bit rate $=128 \mathrm{bit} / \mathrm{s}$, payload size $=1.25 \mathrm{kBytes}$ ).

appends a preamble and then inserts a new set of $N_{p}$ pilot bits before each static block duration $\left(T_{C T}\right)$. Then in phase II it broadcasts the new packet to both Alice and Bob. The raw data were extracted and analyzed in Matlab and the average values are presented in the plot.

Fig. 13 shows the average BER versus transmit power level for unidirectional and bi-directional ANC-CDMA for payload size of $1.25 \mathrm{kBytes}$, fragment size of 125 Bytes, number of pilot bits $N_{P}=32$. We can see that the performance of unidirectional ANC-CDMA is very close to the conventional MU DS-CDMA scheme for a target BER. To achieve a BER of $9 \times 10^{-3}$, a transmit power of about $11.5 \mathrm{~W}$ and $12.6 \mathrm{~W}$ is required with the conventional MU DS-CDMA and ANC-CDMA schemes, respectively. For a penalty of $1.1 \mathrm{~W}$ the proposed scheme can improve the channel utilization by up to $50 \%$ for unidirectional network. As expected, the performance of bi-directional ANCCDMA is slightly worse than unidirectional ANC-CDMA, due to the multi-hop packet propagation and additional channel equalization required for bi-directional transmissions. We can observe that the bi-directional ANC-CDMA performs close to the conventional MU DS-CDMA scheme for a target BER. To achieve a BER of $9 \times 10^{-3}$, a transmit power of about $14.5 \mathrm{~W}$ and $16.1 \mathrm{~W}$ is required with the conventional MU DS-CDMA and ANC-CDMA schemes, respectively. For a penalty of $1.6 \mathrm{~W}$ the proposed scheme can improve the channel utilization by up to $100 \%$ for bi-directional network. We expect the performance to be better in deeper underwater channel where the multipath is expected to be less severe. The simulation model assumes chiplevel synchronization and discrete-time multipath. In real-world experiments, there is no synchronization, but continuous-time multipath. The latter is what makes chip-level synchronization (or lack thereof) irrelevant in practice. At the same time it creates ICI, which is not accounted for in the simulation model.

\section{CONCLUSion AND Future Work}

We have proposed a CDMA-based ANC scheme for unidirectional and bi-directional UW-ASNs, in which two nodes utilize the same spreading code with the intention to improve the underwater acoustic channel utilization. We have developed a receiver that first equalizes the received signal (for the case of bi-directional ANC), then jointly estimates the two multipath faded channels, then suppresses the interfered packet before decoding the information of interest. We have evaluated the BER performance of ANC-CDMA scheme using simulations and actual testbed experiments in a lake. Experiments demonstrate that for a 1-2 dB tradeoff in SNR the proposed scheme can improve the channel utilization by up to $50 \%$ and $100 \%$ for unidirectional and bi-directional networks, respectively compared to the conventional multi-user DS-CDMA scheme.

\section{REFERENCES}

[1] H. Kulhandjian, T. Melodia, and D. Koutsonikolas, "CDMA-based analog network coding through interference cancellation for underwater acoustic sensor networks," in Proc. ACM Int. Conf. WUWNet, Los Angeles, CA, USA, Nov. 2012, pp. 1-8.

[2] I. F. Akyildiz, D. Pompili, and T. Melodia, "Underwater acoustic sensor networks: Research challenges," Ad Hoc Netw. Elsevier, vol. 3, no. 3, pp. 257-279, May 2005.

[3] T. Melodia, H. Kulhandjian, L. Kuo, and E. Demirors, Advances in underwater acoustic networking, in Mobile Ad Hoc Networking: Cutting Edge Directions, 2nd ed. S. Basagni, M. Conti, S. Giordano, and I. Stojmenovic, Eds. Hoboken, NJ, USA: Wiley, 2013. 
[4] M. Stojanovic, Acoustic (underwater) communications, in Encyclopedia of Telecommunications, J. G. Proakis Ed. Hoboken, NJ, USA: Wiley, 2003.

[5] H. Kulhandjian, L. Kuo, T. Melodia, D. A. Pados, and D. Green, "Towards experimental evaluation of software-defined underwater networked systems," in Proc. IEEE UComms, Sestri Levante, Italy, Sep. 2012, pp. 1-9.

[6] S. Zhang, S. Liew, and P. Lam, "Physical layer network coding," in Proc. ACM Int. Conf. MobiCom, Los Angeles, CA, USA, Sep. 2006, pp. 24-29.

[7] S. Katti, S. Gollakota, and D. Katabi, "Embracing wireless interference: Analog network coding," in Proc. ACM SIGCOMM, Kyoto, Japan, Aug. 2007, pp. 397-408.

[8] A. Argyriou and A. Pandharipande, "Cooperative protocol for analog network coding in distributed wireless networks," IEEE Trans. Wireless Commun., vol. 9, no. 10, pp. 3112-3119, Oct. 2010.

[9] M. Dankberg, M. Miller, and M. Mulligan, "Self-interference cancellation for two-party relayed communication," U.S. Patent 5596439 , Jan. 21, 1997.

[10] P. Larsson, N. Johansson, and K.-E. Sunell, "Coded bi-directional relaying," in Proc. IEEE VTC_Spring, Melbourne, Australia, May 2006, vol. 2, pp. 851-855.

[11] B. Rankov and A. Wittneben, "Spectral efficient signaling for halfduplex relay channels," in Proc. IEEE Asilomar Conf. Signals, Syst., Comput., Pacific Grove, CA, USA, Nov. 2005, pp. 1066-1071.

[12] P. Popovski and H. Yomo, "Bi-directional amplification of throughput in a wireless multi-hop network," in Proc. IEEE VTC-Spring, Melbourne, Australia, May 2006, vol. 2, pp. 588-593.

[13] S. Boppana and J. M. Shea, "Overlapped carrier-sense multiple access (OCSMA) in wireless ad hoc networks," IEEE Trans. Mobile Comput., vol. 8, no. 3, pp. 369-383, Mar. 2009.

[14] H. Kulhandjian and T. Melodia, "Modeling underwater acoustic channels in short-range shallow water environments," in Proc. ACM Int. Conf. WUWNet, Rome, Italy, Nov. 2014, pp. 1-5.

[15] "Teledyne-Benthos," Acoust. Modems, Falmouth, MA, USA. [Online]. Available: http://www.benthos.com

[16] H. Gacanin, T. Sjodin, and F. Adachi, "On channel estimation for analog network coding in a frequency-selective fading channel," EURASIP J. Wireless Commun. Netw., vol. 2011, no. 980430, pp. 1-12, 2011.

[17] Z. Wang, J. Huang, S. Zhou, and Z. Wang, "Iterative receiver processing for OFDM modulated physical-layer network coding in underwater acoustic channels," IEEE Trans. Commun., vol. 61, no. 2, pp. 541-553, Feb. 2013.

[18] C. R. Berger, S. Zhou, J. C. Preisig, and P. Willett, "Sparse channel estimation for multicarrier underwater acoustic communication: From subspace methods to compressed sensing," IEEE Trans. Signal Process., vol. 58, no. 3, pp. 1708-1721, Mar. 2010

[19] U. Bhat and T. M. Duman, "Decoding strategies for physical-layer network coding over frequency selective channels," in Proc. IEEE WCNC, Paris, France, Apr. 2012, pp. 12-17.

[20] M. Chen and A. Yener, "Multiuser two-way relaying: Detection and interference management strategies," IEEE Trans. Wireless Commun., vol. 8, no. 8, pp. 4296-4305, Aug. 2009.

[21] M. Rimensberger, Y. E. Sagduyu, M. L. Honig, and W. Utschick, "Comparison of analog and digital relay methods with network coding for wireless multicast," in Proc. IEEE ICC, Dresden, Germany, Jun. 2009, pp. $1-5$.

[22] S. Mao, J. Kim, and J. Lee, "Multi-user analog network coding with spread spectrum," in Proc. IEEE VTC - Spring, Yokohama, Japan, May 2012, pp. 1-5.

[23] H. Kulhandjian and T. Melodia, "A low-cost distributed networked localization and time-synchronization framework for underwater acoustic testbeds," in Proc. IEEE UComms, Sestri Levante, Italy, Sep. 2014, pp. $1-5$.

[24] M. Li, S. N. Batalama, D. A. Pados, T. Melodia, and M. J. Medley, "Cognitive code-division links with blind primary-system identification," IEEE Trans. Wireless Commun., vol. 10, no. 11, pp. 3743-3753, Nov. 2011.

[25] M. Stojanovic and L. Freitag, "Acquisition of direct-sequence spreadspectrum acoustic communication signals," in Proc. IEEE OCEANS, San Diego, CA, USA, Sep. 2003, vol. 1, pp. 279-286.

[26] R. Coates, Underwater Acoustic Systems. New York, NY, USA: Wiley, 1989.

[27] M. Stojanovic, "On the relationship between capacity and distance in an underwater acoustic channel," in Proc. ACM Int. WUWNet, Sep. 2006, pp. 41-47.
[28] H. Kulhandjian, T. Melodia, and D. Koutsonikolas, "Securing underwater acoustic communications through analog network coding," in Proc. IEEE Int. Conf. SECON, Singapore, Jun. 2014, pp. 266-274.

[29] A. J. Laub, Matrix Analysis for Scientists and Engineers. Philadelphia, PA, USA: SIAM, 2005.

[30] K. J. Horadam, Hadamard Matrices and Their Applications. Princeton, NJ, USA: Princeton Univ. Press, 2007

[31] M. Kulhandjian and D. A. Pados, "Uniquely decodable code-division via augmented Sylvester-Hadamard matrices," in Proc. IEEE WCNC, Apr. 2012, pp. 359-363.

[32] B. Steiner and P. Jung, "Optimum and suboptimum channel estimation for the uplink CDMA mobile radio systems with joint detection," Eur. Trans. Telecommun., vol. 5, no. 1, pp. 39-50, Jan./Feb. 1994.

[33] T. C. Yang, "Correlation-based decision-feedback equalizer for underwater acoustic communications," IEEE J. Ocean. Eng., vol. 30, no. 4, pp. 865-880, Oct. 2005.

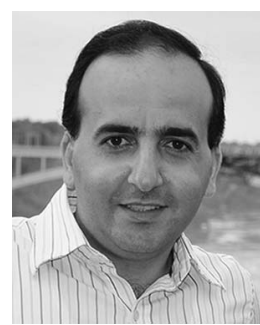

Hovannes Kulhandjian (S'14-M'15) received the B.S. degree (magna cum laude) in electronics engineering from The American University in Cairo, Cairo, Egypt, in 2008, and the M.S. and Ph.D. degrees in electrical engineering from the State University of New York at Buffalo, Buffalo, NY, USA, in 2010 and 2014, respectively.

From December 2014 to July 2015, he was an Associate Research Engineer with the Department of Electrical and Computer Engineering, Northeastern University, Boston, MA, USA. He is currently an Assistant Professor with the Department of Electrical and Computer Engineering, California State University, Fresno, Fresno, CA, USA. His current research interests include digital signal processing, wireless communications and networking, with applications to underwater acoustic communications and networking.

He serves as a member of the Technical Program Committee for ACM and IEEE conferences such as IEEE ICC 2016, WUWNet 2015, GLOBECOM 2015, INFOCOM (Demos/Posters) 2015, and VTC Fall 2015. He received the Outstanding Reviewer Award from Elsevier Ad Hoc Networks.

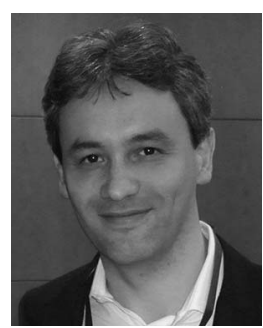

Tommaso Melodia (M'07) received the Ph.D. degree in electrical and computer engineering from the Georgia Institute of Technology, Atlanta, GA, USA, in 2007. He is currently an Associate Professor with the Department of Electrical and Computer Engineering, Northeastern University, Boston, MA, USA. His current research interests include modeling, optimization, and experimental evaluation of networked communication systems, with applications to ultrasonic intrabody networks, cognitive and cooperative networks, multimedia sensor networks, and underwater networks. He was the Technical Program Committee Vice Chair for IEEE GLOBECOM 2013 and the Technical Program Committee Vice Chair for Information Systems for IEEE INFOCOM 2013. He will serve as an Area Chair for IEEE INFOCOM 2016. He serves on the editorial boards of the IEEE Transactions on Mobile Computing, the IEEE TransaCtions on Wireless Communications, the IEEE TRANSACTIONS ON MultimeDIA, and Computer Networks. He is the coauthor of a paper that was recognized as the ISI Fast Breaking Paper in the field of computer science for February 2009 and of an ACM WUWNet 2013 Best Paper Award. His research has been supported by the U.S. National Science Foundation, Air Force Research Laboratory, and the Office of Naval Research, among others. He received the U.S. National Science Foundation CAREER Award.

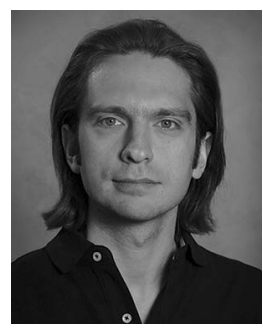

Dimitrios Koutsonikolas (M'11) received the Ph.D. degree in electrical and computer engineering from Purdue University, West Lafayette, IN, USA, in 2010. From September to December 2010, he worked as a Postdoctoral Researcher with Purdue University. He is currently an Assistant Professor with the Department of Computer Science and Engineering at the University at Buffalo, The State University of New York, Buffalo, NY, USA. His research interests include experimental wireless networking and mobile computing. He is a member of ACM and 